\title{
Gene networks in a post-genomic era
}

Ignatieva E.V.

Institute of Cytology and Genetics, SB RAS, Novosibirsk, Russia

Novosibirsk State University, Novosibirsk, Russia

e-mail: eignat@bionet.nsc.ru

Key words: gene networks, feedback, transcription regulatory network, PPIs, SNPs, human diseases

Gene networks are molecular genetic systems that determine phenotypic characteristics of organisms (molecular, biochemical, structural, morphological, behavioral, etc.) using information encoded in the genomes of these organisms [1]. The essential types of structural and functional components of gene networks are: (1) genes; (2) RNA and proteins encoded by genes; (3) low molecular weight components (metabolites, energy transfer molecules, steroid hormones, cations, anions, etc.); (4) negative and positive feedbacks stabilizing the parameters of the gene network at a certain level, or, on the contrary, deviating them from the initial value, providing a transition to a new functional state [2]. Reconstruction of gene networks and deciphering their connectivity structure provide methodological basis for modern systems biology [3]. This review (1) introduces the concepts of Gene networks and related terms used in the post-genomic era (Gene regulatory networks (GRNs), transcription regulatory networks (TRNs), protein-protein interaction (PPI) networks, associative gene networks, etc.); (2) gives an examples of the hierarchical organization of the gene networks (the levels cell, tissue, the whole body, communities of organisms); (3) defines the role of the feedback mechanisms operating at different levels; (4) lists several computer and/or experimental approaches used for gene networks reconstruction; (5) characterizes a number of Internet accessible information resources oriented to humans and animals and containing data on gene networks and their functional modules; (6) refers to computer systems that allow the user to construct molecular-genetic networks using the data automatically extracted from the texts of scientific publications or data on experimentally defined binary interactions between the objects in the network. Applications of network and pathway-based methodology to explore pathogenetic mechanisms underlying human diseases at a systems biology level are also provided.

Acknowledgements: Supported by the State Budgeted Project No. 0324-2019-0040.

\section{References}

1. Ananko E.A.,Aksenovich T.I., Gunbin K.V. et al. Introduction to informational biology and bioinformatics: study guide: eds N.A. Kolchanov, O.V. Vishnevsky, D.P. Furman. V. 3: Chapter 3: Gene Network Theory; Chapter 4: Mapping of genes controlling complex human traits. - Novosibirsk: 2015 (in Russian).

2. Kolchanov N.A. et al. Gene Networks. Vavilov Journal Genetics Breeding. 2013;17(4/2):833-850.

3. Ignatieva E.V. et al. Online resources on gene networks containing human and animal data. Vavilov Journal Genetics Breeding. 2017;21(8):895-902. 